\title{
Geometric and Analytic Views in Existence Theorems for Optimal Control in Banach Spaces. Part 1. Distributed Parameters ${ }^{\star}$
}

\author{
L. CESARI ${ }^{2}$
}

\author{
Dedicated to Professor M. R. Hestenes
}

\begin{abstract}
Existence theorems for optimal control problems in Banach spaces are stated and proved.
\end{abstract}

Key Words, Existence theorems, optimal control, control theory, Banach spaces, distributed parameters.

\section{Introduction}

We state and prove here a new set of existence theorems for problems of optimal control in Banach spaces. These theorems come from work done recently in three different directions. There is the work done by the use of property (Q), Kuratowsky's property (K), and intermediate properties $\left(\mathrm{Q}^{*}\right)$ (Cesari, Refs. 1-4); and Cesari and Cowles, Ref. 5) in connection with various closure and convergence properties of operators in Banach spaces (Cesari and Kaiser, Ref. 6; and Kaiser and Suryanarayana, Refs. 7-8). There is the work done in the line of geometrical properties of the relevant sets [property $(P)$ and variants], which are similar to the familiar ones in Filippov's existence theorem (Cesari, Refs, 9-10). There is finally the work done in the line of Krasnoselski's approach to Nemitsky's operators, based on analytical properties of the relevant functions [Lipschitz-type and growth-type conditions $(F),(G),(H)]$ and no use of seminormality conditions (Cesari and Suryanarayana, Refs. 11-12). Work of Berkovitz (Ref. 13) [on the connections between closure and covergence properties and on the use of properties of the type (F)] is parallel to that mentioned in the first and third of the viewpoints above.

1 This research was partially supported by AFOSR Research Project 71-2122.

${ }^{2}$ Protessor, Department of Mathematics, University of Michigan, Ann Arbor, Michigan. 
In the present paper, we consider problems in a Banach space setting, having mainly in mind multidimensional problems with distributed parameters (though usual one-dimensional problems are well included). In Part 2, we shall consider problems with distributed and boundary controls in Sobolev spaces.

In independent papers, Cesari (Ref. 14) and Suryanararyana (Ref. 15) will prove existence theorems for linear problems without convexity requirements, and Suryanarayana will also prove that convexity and monotonicity-type properties imply property (Q).

\section{Mayer-Type Problems}

2.1. Definitions. We deal here with a topological space $(X, \tau)$, with a Banach space $B$ with norm \|\| , and with metric spaces $(G, \rho)$, $(Y, d),\left(U, d^{\prime}\right)$. In most applications, $X$ will be also a Banach space and $\tau$ its weak topology. We assume that $G$ is also a finite, complete measure space $(G, \alpha, \mu)$ such that the $\sigma$-algebra $\alpha$ contains the Borel sets of $(G, \rho)$ and $\mu$ is regular. Concerning $Y$ and $U$, we assume that they are $\sigma$-compact, that is, the countable union of compact subsets. Let $A$ be any subset in $G \times Y$ such that, for any $t \in G$, the set $A(t)=[y \in Y,(t, y) \in A]$ is nonempty. For every $(t, y) \in A$, let $U(t, y)$ be a given nonempty subset of $U$. Let $f(t, y, u)$ be a given function defined on the set

$$
S=[(t, y, u) \in G \times Y \times U \mid(t, y) \in A, u \in U(t, y)]
$$

with values in $B$, or $f: S \rightarrow B$.

We assume that $A, S$, and the function $f$ satisfy a Carathéodory condition (C) on $G$, that is, given $\epsilon>0$, there is a compact subset $K \subset G$ such that $\mu(G-K)<\epsilon$, the sets

$$
A_{K}=[(t, y) \in A \mid t \in K], \quad S_{K}=[(t, y, u) \in S \mid t \in K]
$$

are closed [in the product topologies of $(G, \rho) \times(Y, d)$ and $(G, \rho) \times$ $(Y, d) \times\left(U, d^{\prime}\right)$, respectively], and that $f$ restricted to $S_{K}$ is continuous (in the weak topology of $B$ ). Thus, there is a set $\bar{T} \subset G, \bar{T} \in \alpha, \mu(\bar{T})=0$, such that, for all $t \in G-\bar{T}$, the sets

$$
A(t)=[y \in Y \mid(t, y) \in A], \quad S(t)=[(y, u) \in Y \times U \mid(t, y, u) \in S]
$$

are closed, and $f(t, \cdot, \cdot)$ is continuous on $S(t)$. We shall denote below by $T_{0}$ some set $T_{0} \subset G, T_{0} \in \alpha, \mu\left(T_{0}\right)=0, \bar{T} \subset T_{0}$.

For any Hausdorff space $H$, we shall denote by $\mu(G, H)$ the set of 
all $\mu$-measurable functions on $G$ with values in $H$. For any Banach space, $B$, we denote by $L_{1}(G, B)$ the set of all strongly integrable $\mu$-measurable functions $\approx$ on $G$ with values in $B$.

For any $p, 1 \leqslant p \leqslant \infty$, we denote by $L_{p}(G, B)$ the set of all $z \in L_{1}(G, B)$ with $\|z(\cdot)\| \in L_{p}\left(G, E^{1}\right)$ and then $\|z\|_{p}$ has the usual definition. Usual conventions hold for $p=\infty$. Thus,

$$
L_{\infty}(G, B) \subset L_{p}(G, B) \subset L_{1}(G, B) \subset \mu(G, B) .
$$

Let $L, M$ be two operators (not necessarily linear) with domains $D(L), D(M) \subset X$ and values in $L_{1}(G, B), \mu(G, Y)$, respectively, and let $X_{0} \subset D(L) \cap D(M)$ be a given nonempty part of their intersection. We shall consider the restrictions of $L$ and $M$ on $X_{0}$, or

$$
L: X_{0} \rightarrow L_{1}(G, B), \quad M: X_{0} \rightarrow \mu(G, Y) .
$$

We shall say that a pair $x, u$ is admissible provided $x \in X_{0}$, $u \in \mu(G, U)$, and

$$
\begin{gathered}
M x(t) \in A(t), \quad u(t) \in U(t, M x(t)), \\
L x(t)=f(t, M x(t), u(t)), \quad \mu \text { a.e. in } G .
\end{gathered}
$$

Then we say that $y=M x \in \mu(G, Y)$ is a state function, and that $u \in \mu(G, U)$ is a strategy, or control function.

We shall consider a given collection $\Omega$ of admissible pairs $x, u$. Then, we shall denote by $\left\{x_{\Omega}\right.$ the collection

$$
\{x\}_{\Omega}=\left\{x \in X_{0},(x, u) \in \Omega \text { for some } u\right\},
$$

and we have $\{x\}_{\Omega} \subset X_{0} \subset X$.

Finally we assume that a lower semicontinuous functional $I$ is defined in $\{x\}_{\Omega}$, that is, $I:\{x\}_{\Omega} \rightarrow E^{1}$, or in a larger class of elements $x \in X_{0}$. We say that the class $\Omega$ is closed (with respect to the Mayer problem under consideration) provided, whenever $\left(x_{k}, u_{k}\right) \in \Omega$ (that is, $\left.x_{k} \in\{x\}_{\Omega}\right), k=1,2, \ldots, x_{k} \rightarrow x$ in $(X, \tau)$ as $k \rightarrow \infty, x \in X_{0}$, and there is some $u \in \mu(G, U)$ such that $x, u$ is admissible, then there is also some $\bar{u} \in \mu(G, U)$ with $(x, \bar{u}) \in \Omega$, thus $x \in\{x\}_{\Omega}$, and

$$
I[x] \leqslant \lim \inf _{k \rightarrow \infty} I\left[x_{k}\right] .
$$

Actually, in most Mayer problems, $I$ is continuous on $\{x\}_{\Omega}$ in the topology $\tau$ of $X$ relatively to $X_{0}$. 


\subsection{Geometric-Type Condition $(P)$ \# A Drastic Reduction of} Property $(Q)$. For $(t, y) \in A$, we shall consider below the sets

$$
Q(t, y)=f(t, y, U(t, y))=[z \in B \mid z=f(t, y, u), u \in U(t, y)] .
$$

We say that the sets $Q(t, y)$ satisfy property (Q) with respect to $y$ at the point $\left(t_{0}, y_{0}\right) \in A$ provided

$$
Q\left(t_{0}, y_{0}\right)=\bigcap_{\epsilon>0} \operatorname{cl} \operatorname{co} \bigcup_{y \in N_{\epsilon}\left(y_{0}\right)} Q\left(t_{0}, y\right)
$$

where

$$
N_{\epsilon}\left(y_{0}\right)=\left[y \in A\left(t_{0}\right), d\left(y, y_{0}\right) \leqslant \epsilon\right] .
$$

We say that, for a given $t_{0} \in G$, the sets $Q(t, y)$ have property $(\mathrm{Q})$ with respect to $y$ in $A\left(t_{0}\right)$ provided the property above holds for all $y_{0} \in A\left(t_{0}\right)$. Sets having property $(Q)$ are necessarily convex and closed.

Property (Q) is an upper semicontinuity property for convex sets (Ref. 3, Part 1) and was proved in Ref. 3, Part 2, to be an extension of usual seminormality conditions for free problems of calculus of variations (see also Ref. 7). Property (Q) is a variant of the analogous Kuratowsky's upper semicontinuity hypothesis for closed sets, or property $(\mathrm{K})$, which in the present terminology [property $(\mathrm{K})$ with respect to $y$ at $\left.\left(t_{0}, y_{0}\right)\right]$ reads

$$
Q\left(t_{0}, y_{0}\right)=\bigcup_{\epsilon>0} \mathrm{cl} \bigcup_{y \in N_{\epsilon}\left(y_{0}\right)} Q\left(t_{0}, y\right) .
$$

Sets having property $(K)$ are necessarily closed.

In Ref. 7, an intermediate property $\left(\mathrm{Q}^{*}\right)$ was introduced. To this end, let $B=B_{1} \times B_{2}$, where $B_{1}$ and $B_{2}$ are both Banach spaces. We say that the sets $Q(t, y)$ satisfy property $\left(\mathrm{Q}^{*}\right)$ relative to $B_{1}$ [or $\left.\left(B_{1}, B_{2}\right)\right]$ at the point $\left(t_{0}, y_{0}\right) \in A$ provided, for any $b_{0}=\left(b_{0}{ }^{1}, b_{0}{ }^{2}\right) \in B=B_{1} \times B_{2}$,

$$
\begin{aligned}
& Q\left(t_{0}, y_{0}\right) \cap\left\{\left(b^{1}, b_{0}{ }^{2}\right) \mid b^{1} \in B_{1}\right\} \\
& \quad=\bigcap_{\epsilon>0} \bigcap_{\delta>0} \mathrm{cl} \operatorname{co}\left[\bigcup_{y \in N_{\epsilon}\left(y_{0}\right)} Q\left(t_{0}, y\right) \cap\left\{\left(b^{1}, b^{2}\right)\|\| b^{2}-b_{0}{ }^{2} \| \leqslant \delta\right] .\right.
\end{aligned}
$$

This condition $\left(\mathrm{Q}^{*}\right)$ reduces to $(\mathrm{Q})$ for $B_{2}=\{0\}$ and to $(\mathrm{K})$ for $B_{1}=\{0\}$. On the other hand, property $\left(Q^{*}\right)$ can be expressed in terms of property (Q) and suitably auxiliary sets, as it was shown in Ref. 7.

In the following, for any given $N>0$, we denote by $V(0, N)$ the subset of the Banach space $B$ defined by $[z \in B \mid\|z\| \leqslant N]$.

For sets $Q(t, y)$ which are closed and convex, the following geometric type conditions $(\mathrm{P})$ represent a drastic reduction of property $(\mathrm{Q})$.

We say that the sets $Q(t, y), y \in A(t)$, satisfy condition (P1) if 
there is a bounded $\mu$-measurable function $p(t), t \in G, p \in \mu(G, B)$, $p: G \rightarrow B,\|p(t)\|_{B} \leqslant \sigma$ for all $t \in G$, such that $p(t) \in Q(t, y)$ for all $t \in G-T_{0}, y \in A(t)$.

We say that the sets $Q(t, y), y \in A(t)$, also satisfy condition (P2) if, for every $t \in G-T_{0}$ and $N>\sigma,(\sigma$ as in P1), the convex closed equibounded sets $Q(t, y) \cap V(0, N)$ satisfy property (Q) with respect to $y$ in $A(t)$.

Property (P1) is certainly satisfied if, for instance, all sets $Q(t, y)$ contain the origin of $B$ [or all sets $U(t, y)$ contain the origin of $U$ and $f(t, y, 0)=0]$. This condition is usually satisfied in applications.

Concerning (P2), we only note that, for $B=E^{r}, Y=E^{s}, U=E^{m}$, the sets $Q(t, y) \cap V(0, N)$ are convex, equibounded, and compact, and the less demanding property $(\mathrm{K})$ can well replace property $(\mathrm{Q})$ in $(\mathrm{P} 2)$. Moreover, property $(\mathrm{P} 2)$ is then certainly satisfied if $|f(t, y, u)| \rightarrow \infty$ as $|u| \rightarrow+\infty$ uniformly on any bounded subset of $A(t)$ [as a consequence of the mere continuity of $f$ on $S(t)$ for $t \in G-T_{0}$ ]. For convex equibounded compact sets in Euclidean spaces, property $(Q)$ is equivalent to upper semicontinuity by set inclusion, and thus $(\mathrm{P} 2)$ is similar to the analogous requirement in Filippov's existence theorem.

2.3. Properties of Operators. Let $(X, \tau)$ be any topological space, $Z$ a Banach space, $X_{0}$ a subset of $X$, and $A: X_{0} \rightarrow Z$ a given operator, not necessarily linear.

The operator $A$ is said to have the strong (weak) closure property on $X_{0}$ with respect to $(X, \tau)$ provided $x_{k} \in X_{0}, k=1,2, \ldots, x_{k} \rightarrow x$ in $(X, \tau)$ as $k \rightarrow \infty, x \in X_{0}, A x_{k} \rightarrow y$ strongly (weakly) in $Z$ implies that $A x=y$.

The operator $A$ is said to have the strong (weak) closed graph property on $X_{0}$ with respect to $(X, \tau)$ provided $x_{k} \in X_{0}, k=1,2, \ldots$ $x_{k} \rightarrow x$ in $(X, \tau)$ as $k \rightarrow \infty, x \in X, A x_{k} \rightarrow y$ strongly (weakly) in $Z$ implies that $x \in X_{0}$ and $A x=y$.

We mention here that, whenever $X_{0}=X$, closure property and closed graph property coincide. In our previous paper (Ref. 5), it was always assumed that $X_{0}=X$; and, in all examples in Ref. 5 concerning some Sobolev space $X$, the closure property was satisfied.

The operator $A$ is said to have the strong(weak) convergence property on $X_{0}$ with respect to $(X, \tau)$ provided $x_{k} \in X_{0}, k=1,2, \ldots, x_{k} \rightarrow x$ in $(X, \tau)$ as $k \rightarrow \infty$, implies that there is a subsequence $\left[k_{s}\right]$ such that $A x_{k_{0}}, s=1,2, \ldots$, is strongly (weakly) convergent in $Z$.

If $(G, \alpha, \mu)$ is a finite measure space and $Z=\mu(G, M)$ is the space of all $\mu$-measurable functions over $G$ with values in a metric space $(M, d)$ and $A: X_{0} \rightarrow Z$, then we can define the convergence in measure property on $X_{0}$ with respect to $(X, \tau)$. 
If $(G, \alpha, \mu)$ is as above and $Z=L_{p}(G, B)$ is the space of all $p$-integrable functions over $G$ with values in a Banach space $B$, and $A$ : $X_{0} \rightarrow Z$, then we can define a strong (weak) $L_{p}$-convergence property on $X_{0}$ with respect to $(X, \tau)$.

If $X_{00} \subset X_{0} \subset X$ and, in the properties above we assume that $x_{k} \in X_{00}, k=1,2, \ldots$ (but $x$ is still required to be in $X_{0}$ ), then we say that the properties above hold relatively to $X_{00}$.

If $x_{k} \in X_{00}, x_{k} \rightarrow x$ in $(X, \tau)$ implies that $x \in X_{0}$, then we say that the closure of $X_{00}$ in $(X, \tau)$ is contained in $X_{0}$, or briefly $\mathrm{cl} X_{00} \subset X_{0}$ [with respect to the space $(X, \tau)$ ]. This property is trivial if $X_{0}=X$.

If $\Omega$ is a class of admissible pairs $x, u$ with $x \in\{x\}_{\Omega} \subset X_{0} \subset X$, $u \in \mu(G, U)$, we say that $f$ has the strong (weak) convergence property in $L_{p}(G, B)$ for some $p, 1 \leqslant p \leqslant \infty$, provided $\left(x_{k}, u_{k}\right) \in \Omega, k=$ $1,2, \ldots, x_{k} \rightarrow x$ in $(X, \tau)$ as $k \rightarrow \infty$ implies that, for

$$
z_{k}(t)=f\left(t, x_{k}(t), u_{k}(t)\right), \quad t \in G, k=1,2, \ldots,
$$

we have $z_{k} \in L_{p}(G, B)$, and there is a subsequence $\left[k_{s}\right]$ such that $z_{k_{s}} \rightarrow z$ strongly (weakly) in $L_{p}(G, B)$ as $s \rightarrow \infty$.

In some of the alternate conditions in Section 2.3 with $Y$ and $U$ Banach spaces, we shall need also the following very mild continuity condition $(\Gamma)$ on $f$ : there are a constant $\gamma>0$, a $\mu$-measurable function $\Gamma(t) \geqslant 0, t \in G, \Gamma \in \mu\left(G, E^{1}\right)$, and a monotone nondecreasing function $h(\xi) \geqslant 0,0 \leqslant \xi<\infty, h(0+)=0$, such that, for all $\left(t, y_{1}, u\right)$, $\left(t, y_{2}, u\right) \in S$, with $\left\|y_{1}\right\|_{Y},\left\|y_{2}\right\|_{Y},\|u\|_{U} \leqslant \gamma$, we have

$$
\left\|f\left(t, y_{1}, u\right)-f\left(t, y_{2}, u\right)\right\|_{B} \leqslant \Gamma(t) h\left(\left\|y_{1}-y_{2}\right\|_{Y}\right) .
$$

This condition $(I)$ is a consequence of condition (C) if $Y=E^{s}, U=E^{m}$ are both Euclidean spaces.

Concerning the measure space $G$ and the Banach space $B$, we shall need in some of our alternate conditions one or another of the following properties:

(R1) Any sequence $z_{k}(t), t \in G, z_{k} \in L_{\infty}(G, B), k=1,2, \ldots$, with $\left\|z_{k}\right\|_{\infty} \leqslant N$ for all $k=1,2, \ldots$, possesses a subsequence which is weakly convergent in $L_{\infty}(G, B)$ [the same property for $z_{k} \in L_{p}(G, B), 1<p<\infty$, will be denoted by $(\mathrm{R} 1)_{p}$ ].

(R2) Any sequence $z_{k}(t), t \in G, z_{k} \in L_{1}(G, B), k=1,2, \ldots$, which is weakly convergent in $L_{1}(G, B)$ is equiabsolutely integrable in $G$.

(R3) Any sequence $z_{k}(t), t \in G, z_{k} \in L_{1}(G, B), k=1,2, \ldots$, which is equiabsolutely integrable in $G$ (and thus also equibounded in $L_{1}$ ), possesses a subsequence which is weaklt convergent in $L_{1}(G, B)$.

For $B$ a Euclidean space, (R1) is well known, and (R3) is a restate- 
ment of the Dunford-Pettis theorem for finite measure spaces. For $1<p<\infty,(\mathrm{R} 1)_{p}$ is true for any separable reflexive Banach space $B$.

2.4. Existence Statement for Mayer Problems. Let $A, S, f$ satisfy condition (C). Let us assume that $\Omega$ is not empty and closed, that $\{x\}_{\Omega}$ is sequentially relatively compact in $(X, \tau)$, and that $l[x]$ is bounded below on $\{x\}_{\Omega}$. Let us assume that both operators $L$ and $M$ have the closure property in $X_{0}$, and that at least one of them has the closed graph property in $X_{0}$. Then the functional $I[x]$ has an absolute minimum in $\{x\}_{\Omega}$, provided any of the following alternate assumptions below holds.

Alternate Hypotheses. We list here only a few of the possible alternate hypotheses. Requirements $(\mathrm{AQ})$ to $\left(\mathrm{AQ}^{* *}\right)$ use variants of property $(\mathrm{Q})$; requirements $(\mathrm{AP})$ to $\left.(\mathrm{AP})^{* *}\right)$ are of the geometric type; requirements $\left(F_{p}\right),\left(G_{p q}\right),\left(H_{q}\right)$ are analytic conditions.

(AQ) $B$ Banach, $Y, U$ metric. We assume that (i) $\{x\}_{\Omega} \subset X_{0} \subset X$; (ii) $L: X_{0} \rightarrow L_{1}(G, B)$ has weak convergence property relatively to $\{x\}_{\Omega}$; (iii) $M: X_{0} \rightarrow \mu(G, Y)$ has convergence in measure property relatively to $\{x\}_{\Omega}$. We assume finally that, for every $t \in G-T_{0}$, the sets $Q(t, y)$ are closed, convex, and have property (Q) with respect to $y$ in $A(t)$.

Alternatively, if we assume that $f$ has weak convergence property in $L_{1}(G, B)$, then (ii) can be deleted.

(AK) $B$ Banach, $Y, U$ metric. We assume that (i) $\{x\}_{\Omega} \subset X_{0} \subset X$; (ii) $L: X_{0} \rightarrow L_{1}(G, B)$ has strong convergence property relatively to $\{x\}_{\Omega}$; (and iii) $M: X_{0} \rightarrow \mu(G, Y)$ has convergence in measure property relatively to $\{x\}_{\Omega}$. We assume finally that, for every $t \in G-T_{0}$, the sets $Q(t, y)$ are closed and have property (K) with respect to $y$ in $A(t)$.

Alternatively, if we assume that $f$ has strong convergence property in $L_{7}(G, B)$, then (ii) can be deleted.

$\left(\mathrm{AQ}^{*}\right) \quad B=B_{1} \times B_{2}$ Banach, $Y, U$ metric; $L=L_{1} \times L_{2}$, $f=\left(f_{1}, f_{2}\right), f_{i}: S \rightarrow B_{i}, L_{i}: X_{0} \rightarrow L_{1}\left(G, B_{i}\right), i=1,2, M: X_{0} \rightarrow \mu(G, Y)$, and equation $L x=f$ is replaced by $L_{i} x(t)=f_{i}(t, M x(t), u(t)), t \subset G$, $i=1,2$. We assume that (i) $\{x\}_{\Omega} \subset X_{0} \subset X$; (ii) $L_{1}$ has weak convergence property; (iii) $L_{2}$ has strong convergence property; and (iv) $M$ has convergence in measure property (all $L_{1}, L_{2}, M$ relatively to $\{x\}_{\Omega}$ ). We assume finally that, for every $t \in G-T_{0}$, the sets $Q(t, y)$ satisfy the property $\left(\mathrm{Q}^{*}\right)$ with respect to $y$ in $A(t)$ and relatively to $\left(B_{1}, B_{2}\right)$ (see definition of property $\left(\mathrm{Q}^{*}\right)$ in Section 2.2). Here we assume that all operators $L_{1}, L_{2}, M$ have the closure property in $X_{0}$, and that at least one of them has the closed graph property in $X_{0}$. 
The condition $\left(\mathrm{Q}^{*}\right)$ reduces to $(\mathrm{Q})$ for $B_{2}=\{0\}$, and to $(\mathrm{K})$ for $B_{1}=\{0\}$.

Alternatively, if we assume that $f_{1}$ has weak convergence property in $L_{1}\left(G, B_{1}\right)$, then (ii) can be deleted.

Another combination of the various hypotheses, is, for instance, as follows.

(AQ**) $B=B_{1} \times B_{2} \times B_{3} \times B_{4}$ Banach, $Y, U$ metric; $L=$ $L_{1} \times L_{2} \times L_{3} \times L_{4}, f=\left(f_{1}, f_{2}, f_{3}, f_{4}\right), f_{i}: S \rightarrow B_{i}, L_{i}: X_{0} \rightarrow L_{1}\left(G, B_{i}\right)$, $i=1,2,3,4, M: X_{0} \rightarrow \mu(G, Y)$ and equation $L x=f$ is replaced by $\left.L_{i} x(t)=f_{i}(t, M x(t)), u(t)\right), i=1,2,3,4$. Spaces $B_{2}, B_{3}, B_{4}$ may be trivial, but $B_{1}$ is not. We assume that $L_{2}$ has weak convergence property, $L_{3}$ has closure in measure property, $L_{4}$ and $M$ have convergence in measure property (all relatively to $\{x\}_{\Omega}$ ). We assume that $f_{1}$ has the weak convergence property in $L_{1}\left(G, B_{1}\right)$, and that $f_{3}(t, y)$ is independent of $u$. Finally, we assume that, for every $t \in G-T_{0}$, the sets $Q(t, y)$ satisfy property $\left(\mathrm{Q}^{*}\right)$ with respect to $y$ in $A(t)$ relatively to $\left(B_{1} \times B_{2}, B_{3} \times B_{4}\right)$. Here we assume that all operators $L_{1}, L_{2}, L_{3}, L_{4}, M$ have the closure property in $X_{0}$, and that at least one of them (is not trivial and) has the closed graph property in $X_{0}$.

(AP) $B$ Banach with properties (R1), (R2); $Y, U$ metric. We assume that (i) $\{x\}_{\Omega} \subset X_{0} \subset X$; and (ii) $L: X_{0} \rightarrow L_{1}(G, B)$ has weak convergence property relatively to $\{x\}_{\Omega} ; M: X_{0} \rightarrow \mu(G, Y)$ has convergence in measure property relatively to $\{x\}_{\Omega}$. We assume that the sets $Q(t, y)$ are closed and convex, and satisfy geometric type conditions (P1), (P2).

Alternatively, if we assume that $f$ has weak convergence property in $L_{1}(G, B)$, then (ii) can be deleted.

$\left(\mathrm{AP}^{*}\right) \quad B=B_{1} \times B_{2}$ as $\left(\mathrm{AQ}^{*}\right), B_{1}$ with properties (R1), (R2), $Y, U$ metric; $L=L_{1} \times L_{2}, f=\left(f_{1}, f_{2}\right), f_{i}, L_{i}, M$ as in (AQ $\left.{ }^{*}\right)$. Properties (i) to (iv) as in (AQ*). We assume properties (Pl), as in Section 2.2, with the sets $Q(t, y) \cap V(0, N)$ having property $\left(\mathrm{Q}^{*}\right)$ with respect to $y$ in $A(t)$ relatively to $\left(B_{1}, B_{2}\right)$.

Again, if $f_{1}$ has weak convergence property in $L_{1}\left(G, B_{1}\right)$, then (ii) can be deleted.

( $\left.\mathrm{AP}^{* *}\right) \quad B=B_{1} \times B_{2} \times B_{3} \times B_{4}$ as in $\left(\mathrm{AQ}^{* *}\right), B_{1}$ with properties (R1), (R2), and the same other properties for $L_{i}, f_{i}, M$ as in $\left(\mathrm{AQ}^{* *}\right)$. We assume properties $(\mathrm{P} 1)$, as in Section 2.2, with the sets $Q(t, y) \cap V(0, N)$ having property $\left(\mathrm{Q}^{*}\right)$ with respect to $y$ in $A(t)$ relatively to $\left(B_{1} \times B_{2}, B_{3} \times B_{4}\right)$.

$\left(\mathrm{F}_{13}\right), 1 \leqslant p \leqslant \infty$. Here, both $B$ and $Y$ are Banach spaces, $U$ metric. (i) $\{x\}_{\Omega} \subset X_{0} \subset X$; (ii) $L: X_{0} \rightarrow L_{1}(G, B)$ has weak convergence property relatively to $\{x\}_{\Omega}$; and (iii) $M: X_{0} \rightarrow L \zeta(G, Y)$ has strong 
$L_{p}$-convergence property relatively to $\{x\}_{\Omega}$. The sets $U(t)$ depend on $t$ only, the sets $Q(t, y)$ are closed and convex. If $1 \leqslant p<\infty$, there are constants $c, \gamma, \zeta_{0}, p^{\prime}$ constants with $c \geqslant 0, \zeta_{0} \geqslant 0,0<\gamma \leqslant p$, $p^{\prime}=p(p-\gamma)^{-1}$ if $0<\gamma<p, p^{\prime}=\infty$ if $\gamma=p$, and functions $F(t) \geqslant 0$, $t \in G, F \in L_{p^{1}}\left(G, E^{1}\right), h(\zeta), \quad 0 \leqslant \zeta<\infty, h$ monotone nondecreasing, $h\left(0^{+}\right)=0, h(\zeta) \leqslant c|\zeta|^{\gamma}$ for $\zeta \geqslant \zeta_{0}$, such that, for all $\left(t, y_{1}, u\right),\left(t, y_{2}, u\right) \in S$, $t \in G-T_{0}$, we have

$$
\left\|f\left(t, y_{1}, u\right)-f\left(t, y_{2}, u\right)\right\|_{B} \leqslant F(t) h\left(\| y_{1}-\left.y_{2}\right|_{Y}\right) .
$$

For $p=\infty$, this same relation holds for some $F(t) \geqslant 0, t \in G$, $F \in L_{1}\left(G, E^{1}\right), \quad h(\zeta), \quad 0 \leqslant \zeta<\infty, h$ monotone nondecreasing, with $h\left(0^{+}\right)=0$. Requirement (1) is slightly more restrictive than $(\Gamma)$.

$\left(\mathrm{G}_{p q}\right), 1 \leqslant p, q \leqslant \infty$. Here, $B, Y, U$ are Banach spaces, strategies are elements $u \in L_{q}(G, U)$ with $\|u\|_{q} \leqslant N$ for some $N>0$, and (i), (ii), (iii) hold as in $\left(F_{p}\right)$. The sets $U(t)$ depend on $t$ only, the sets $Q(t, y)$ are closed and convex. Also, $f$ satisfies continuity property $(T)$. Moreover, for $1 \leqslant p, q<\infty$, there are constants $c, c^{\prime} \geqslant 0,0<\alpha \leqslant p, 0<\beta \leqslant q$, and a function $F(t) \geqslant 0, t \in G, F \in L_{1}\left(G, E^{1}\right)$ such that, for all $\left(t, y_{1}, u\right)$, $\left(t, y_{2}, u\right) \in S, t \in G-T_{0}$, we have

$$
\left\|f\left(t, y_{1}, u\right)-f\left(t, y_{2}, u\right)\right\|_{B} \leqslant F(t)+c\left(\left.\left\|\left.y_{1}\right|_{Y} ^{p-\alpha}+\right\| y_{2}\right|_{Y} ^{p-\alpha}\right)+c^{\prime}\|u\|_{U}^{Q-\beta} .
$$

For $p=\infty, 1 \leqslant q<\infty$, there are constants $c^{\prime} \geqslant 0,0<\beta \leqslant q$, and functions $F$ as above, and $\sigma(\zeta) \geqslant 0,0 \leqslant \zeta<\infty, \sigma$ monotone nondecreasing such that

$$
\left.\left\|f\left(t, y_{1}, u\right)-f\left(t, y_{2}, u\right)_{B}^{\|_{B}} \leqslant F(t) \sigma\left(\left\|y_{1}\right\|_{Y}+: \mid y_{2} \|_{Y}\right)+c^{\prime}\right\| u\right|_{i U} ^{Q-\beta} .
$$

If $q=\infty$, then a term $\sigma\left(\|u\|_{U}\right)$ replaces $c^{\prime}\|u\|_{U}^{q-\beta}$ in the relations above.

Note that $\sigma$ is not required to satisfy $\sigma\left(0^{+}\right)=0$ and, therefore, these analytic conditions on $f$ are essentially growth properties.

$\left(\mathrm{H}_{q}\right), 1 \leqslant q \leqslant \infty$. Here, $B, U$ are Banach spaces, $Y$ metric, strategies are elements $u \in L_{q}(G, U)$ with $\|u\|_{q} \leqslant N$, for some $N \geqslant 0$, and (i) $\{x\}_{\Omega} \subset X_{0} \subset X$; (ii) $L: X_{0} \rightarrow L_{1}(G, B)$ has weak convergence property; and (iii) $M: X_{0} \rightarrow \mu(G, Y)$ has convergence in measure property. Also, $f$ satisfies continuity property $(T)$. Moreover, if $1 \leqslant q<\infty$, there are constants $c^{\prime} \geqslant 0,0<\beta \leqslant q$, and a function $F(t) \geqslant 0, t \in G, F \in L_{1}\left(G, E^{1}\right)$ such that, for all $\left(t, y_{1}, u\right),\left(t, y_{2}, u\right) \in S$, we have

$$
\left\|f\left(t, y_{1}, u\right)-f\left(t, y_{2}, u\right)\right\|_{B} \leqslant F(t)+c^{\prime}\|u\|_{Z}^{q-\beta} .
$$


For $q=\infty$, there are functions $F$ as above and $\sigma(\zeta) \geqslant 0,0 \leqslant \zeta<\infty$, $\sigma$ monotone nondecreasing such that

$$
\left\|f\left(t, y_{1}, u\right)-f\left(t, y_{2}, u\right)\right\|_{B} \leqslant F(t) \sigma\left(\|u\|_{U}\right) .
$$

Note that $\sigma$ is not required to satisfy $\sigma\left(0^{+}\right)=0$ and, therefore, these analytic conditions are essentially growth conditions.

2.5. Case of $f$ Linear in $\boldsymbol{u}$. We assume here that $U(t)$ depends on $t$ only, that $B, Y, U$ are Banach spaces, and that $f(t, y, u)$ is linear in $u$, that is, of the form

$$
f(t, y, u)=C(t, y) u+D(t, y) .
$$

Thus, $D: A \rightarrow B, C: A \rightarrow \mathscr{L}=\mathscr{L}(U, B)$, the Banach space of all bounded linear operators from $U$ to $B$. The statement in Section 2.4 still holds under each of the alternate hypotheses $(A Q)$ to $\left(\mathrm{H}_{\infty}\right)$.

The following assumptions on $C, D$ imply the corresponding assumptions on $f$.

Carathéodory's condition (C) on $f$ certainly holds if we assume:

$\left(\mathrm{C}^{\prime}\right)$ Given $\epsilon>0$, there is some compact subset $K$ of $G$ such that $\mu(G-K)<\epsilon$, the sets

$$
\begin{gathered}
A_{K}=[(t, y) \mid t \in K, y \in A(t)] \subset G \times Y, \\
S_{K}=[(t, y, u) \mid t \in K, y \in A(t), u \in U(t)] \subset G \times Y \times U
\end{gathered}
$$

are closed, and $D(t, y), C(t, y)$ are continuous on $A_{K}$.

Condition $(I)$ of Section 2.3 on $f$ certainly holds if we assume:

$\left(\Gamma^{\prime}\right)$ There is a constant $\gamma>0$, a $\mu$-measurable function $\Gamma(t) \geqslant 0$, $t \in G, \Gamma \in \mu\left(G, E^{1}\right)$, and a monotone nondecreasing function $h(\zeta) \geqslant 0$, $0 \leqslant \zeta<\infty, h\left(0^{+}\right)=0$, such that, for all $\left(t, y_{1}\right),\left(t, y_{2}\right) \in A$ with $\left\|y_{1}\right\|$, $\left\|y_{2}\right\| \leqslant \gamma$, we have

$$
\left\|C\left(t, y_{1}\right)-C\left(t, y_{2}\right)\right\|_{\mathscr{L}}, \quad\left\|D\left(t, y_{1}\right)-D\left(t, y_{2}\right)\right\|_{B} \leqslant \Gamma(t) h\left(\left\|y_{1}-y_{2}\right\|_{Y}\right) .
$$

If $Y=E^{s}, U=E^{m}$, then (C') implies $\left(\Gamma^{\prime}\right)$.

The statement in Section 2.4 holds in the present situation, with $\left(\mathrm{C}^{\prime}\right)$ replacing $(\mathrm{C})$ and the corresponding alternate assumptions $\left[\left(\Gamma^{\prime}\right)\right.$ replacing $(T)$ whenever needed].

Conditions $\left(\mathrm{G}_{p q}\right)$ to $\left(\mathrm{H}_{\infty}\right)$ can be replaced by analogous conditions, say $\left(\mathrm{G}_{p q}^{\prime}\right)$ to $\left(\mathrm{H}_{\infty}{ }^{\prime}\right)$ on $C$ and $D$. For instance, $\left(\mathrm{G}_{p q}^{\prime}\right)$ is the same as $\left(G_{p q}\right)$, with the following assumption replacing (2): 
$\left(\mathrm{G}_{p q 2}^{\prime}\right)$ There are constants $c \geqslant 0,0<\alpha \leqslant p$, and a function $F(t) \geqslant 0, t \in G, F \in L_{1}\left(G, E^{1}\right)$, such that, for all $\left(t, y_{1}\right),\left(t, y_{2}\right) \in A$, we have

$$
\|C(t, y)\|_{\mathscr{L}} \leqslant c, \quad\left\|D\left(t, y_{1}\right)-D\left(t, y_{2}\right)\right\|_{B} \leqslant F(t)+c\left(\mid y_{1}\left\|_{Y}^{p-\alpha}+\right\| y_{2} \|_{Y}^{p-\alpha}\right) .
$$

Condition $\left(\mathrm{G}_{p q}^{\prime}\right)$ on $C, D$ implies condition $\left(\mathrm{G}_{p q}\right)$ on $f$.

Finally, new alternate conditions can now be stated fot $f$ linear in $u$, as for instance, the following one:

$\left(\mathrm{G}_{p q}^{\prime \prime}\right), 1<p, q \leqslant \infty$. Here, $B, Y, U$ are Banach spaces, strategies in $\Omega$ are elements $u \in L_{q}(G, U)$ with $\|u\|_{q} \leqslant N$ for some $N \geqslant 0, L$ : $X_{0} \rightarrow L_{1}(G, B)$ has the weak closure property, and $M: X_{0} \rightarrow L_{p}(G, Y)$ has the strong $L_{p}$-convergence property, relatively to $\{x\}_{\Omega}$. The sets $U(t)$ depend on $t$ only, and the sets $Q(t, y)$ are closed and convex. Here, $f=C(t, y) u+D(t, y)$, with $C, D$ satisfying conditions $\left(\mathcal{C}^{\prime}\right)$ and $\left(\Gamma^{\prime}\right)$. Moreover, if $1<p<\infty$, there are constants $c \geqslant 0,0<\alpha \leqslant p$, $p^{\prime}=p(p-\alpha)^{-1}$ if $0<\alpha<p, p^{\prime}=\infty$ if $\alpha=p$, and functions $F(t) \geqslant 0$, $F_{0}(t) \geqslant 0, t \in G, F \in L_{p}\left(G, E^{1}\right), F_{0} \in L_{p^{\prime}}\left(G, E^{x}\right)$, such that, for all $(t, y)$, $\left(t, y_{1}\right),\left(t, y_{2}\right) \in A$, we have

$$
\begin{aligned}
& \|C(t, y)\|_{\mathscr{L}} \leqslant c, \quad \| D\left(t, y_{1}\right)-\left.D\left(t, y_{2}\right)\right|_{B} \leqslant F(t)+c\left(\left\|y_{1}\right\|_{Y}^{(t-\alpha}+\left\|y_{2}\right\|_{Y}^{p-\alpha}\right), \\
& \|D(t, y)\|_{B} \leqslant F_{0}(t)+c\|y\|_{Y}^{w^{*}} .
\end{aligned}
$$

Here, we assume that property (R1) $)_{Q}$ holds for $G, U$, and $(\mathrm{R} 1)_{p^{\prime}}$ for $G, Y$. If $p=\infty$, then we assume that there are functions $F(t) \geqslant 0$, $t \in G, F \in L_{\infty}\left(G, E^{1}\right), \sigma(\zeta), 0 \leqslant \zeta<\infty, \sigma$ monotone nondecreasing, we assume that relations above hold with second members $c, F(t) \sigma\left(\left\|y_{1}\right\|_{Y}+\right.$ $\left.\left\|y_{2}\right\|_{Y}\right)$, and $F(t) \sigma\left(\|y\|_{Y}\right)$, respectively, and we assume property $(\mathrm{R} 1)_{\infty}$ for $G, Y$.

Note that here the conditions imply that $f$ has the weak convergence property in $L_{1}(G, B)$.

2.6. Proof of Statements in Sections 2.4 and 2.5. Since $I[x]$ is bounded below on $\{x\}_{\Omega}$, the infimum $i$ of $I[x]$ on $\{x\}_{\Omega}$ is finite, and we take a sequence $x_{k} \in\left\{x_{\delta_{2}}, k=1,2, \ldots\right.$, with $I\left[x_{k}\right] \rightarrow i$ as $k \rightarrow \infty$. Then, for each $k$, there is some element $u_{k}$ such that $\left(x_{k}, u_{k}\right) \in \Omega$. By compactness hypothesis of $\{x\}_{\Omega}$, there is a subsequence, say [k], such that $x_{k} \rightarrow x$ in $(X, \tau)$ and thus $x \in X$. Under any of the alternate assumptions, we can derive that $x \in X_{0}$, and that there is a subsequence, say still [k], such that $L x_{k} \rightarrow L x$ weakly in $L_{1}(G, B)$, and moreover $M x_{k} \rightarrow M x$ 
in the corresponding mode as indicated in the assumption. The existence of an element $u$ such that $x, u$ is admissible is a consequence of closure theorems. Under assumptions $(\mathrm{AQ}),\left(\mathrm{AQ}^{*}\right),\left(\mathrm{AQ}^{* *}\right)$, we use the closure theorem proved in Ref. 8 in terms of Banach spaces. Under assumptions $(\mathrm{AP}),\left(\mathrm{AP}^{*}\right),\left(\mathrm{AP}^{* *}\right)$, we use closure theorems proved in Ref. 9 in terms of Euclidean spaces, but the proofs for Banach spaces are the same under the specific hypotheses $\left(\Gamma^{\prime}\right),(\mathrm{R} 123)$. Under assumptions $(\mathrm{F}),(\mathrm{G}),(\mathrm{H})$, we use closure theorems proved in Ref. 11 in terms of Euclidean spaces, but the proofs in Banach spaces again are the same. By the closure property of $\Omega$, there is $(x, \bar{u}) \in \Omega$ with $I[x] \leqslant i$. Thus, $x \in\{x\}_{\Omega}, I[x] \geqslant i$, and, hence, $I[x]=i$. The statements in Section 2.5 follow easily.

\section{Lagrange-Type Problems}

3.1. Definitions. As in Section 2.1, we deal with a topological space $(X, \tau)$, with a Banach space $B$ with norm \|\| , and with metric spaces $(G, \rho),(Y, d),\left(U, d^{\prime}\right)$. Again, $G$ is also a measure space $(G, \alpha, \mu)$ as detailed in Section 2.1. Also, $Y, U, A, A(t), U(t, y), S$ are as in Section 2.1, and besides $f: S \rightarrow B$ we have here a real-valued function $f_{0}(t, y, u)$ on $S$, or $f_{0}: S \rightarrow E^{1}$. We assume that $A, S$, and both $f_{0}$ and $f$ satisfy a Carathéodory property (C). For every $(t, y) \in A$, we consider here the set

$$
\tilde{Q}(t, y)=\left[\left(z^{0}, z\right) \in E^{1} \times B \mid z^{0} \geqslant f_{0}(t, y, u), z=f(t, y, u), u \in U(t, y)\right] .
$$

As in Section 2.1, $L$ and $M$ denote operators, not necessarily linear, $L: X_{0} \rightarrow L_{1}(G, B), M: X_{0} \rightarrow \mu(G, Y)$, with $X_{0} \subset D(L) \cap D(M) \subset X$. We say that a pair $x, u$ is admissible provided $x \in X_{0}, u \in \mu(G, U)$,

$$
\begin{aligned}
M x(t) \in A(t), \quad u(t) \in U(t, M x(t)), \quad L x(t) & =f(t, M x(t), u(t)), \\
\mu \text {-a.e. in } G, \quad \text { and } f_{0}(\cdot, M x(\cdot), u(\cdot)) & \in L_{1}\left(G, E^{1}\right),
\end{aligned}
$$

and thus the integral

$$
I[x, u]=\int_{G} f_{0}(t, M x(t), u(t)) d \mu
$$

has a finite value for every admissible pair $(x, u)$.

We shall consider a given class $\Omega$ of admissible pairs $(x, u)$; and thus $I$ is a functional defined on $\Omega$, or $I: \Omega \rightarrow E^{1}$.

As in Section 2.1, we denote by $\{x\}_{\Omega}$ the collection $\{x\}_{\Omega}=\left\{x \in X_{0}\right.$, $(x, u) \in \Omega$ for some $u\}$, so that $\{x\}_{\Omega} \subset X_{0} \subset X$. We shall say that the class $\Omega$ is closed (with respect to the Lagrange problem under consideration) 
provided, whenever $\left(x_{k}, u_{k}\right) \in \Omega, k=1,2, \ldots, x_{k} \rightarrow x$ in $(X, \tau)$ as $k \rightarrow \infty$, $x \in X_{0}$, and there is some $u \in \mu(G, U)$ such that $(x, u)$ is admissible, then there is also some $\bar{u} \in \mu(G, U)$ such that

$$
(x, \bar{u}) \in \Omega, \quad I[x, \bar{u}] \leqslant \lim \inf I\left[x_{k}, u_{k}\right] .
$$

In some of our statements below, we shall need one or another of the following growth conditions:

$(\psi)$ Given $\epsilon>0$ there is $\psi_{\varepsilon}(t) \geqslant 0, t \in G, \psi_{\varepsilon} \in L_{1}\left(G, E^{1}\right)$ such that $\|f(t, y, u)\|_{B} \leqslant \psi_{\epsilon}(t)+\epsilon f_{0}(t, y, u)$ for all $(t, y, u) \in S$.

$(\alpha)$ There is $\psi(t) \geqslant 0, t \in G, \psi \in L_{1}\left(G, E^{1}\right)$ such that $f_{0}(t, y, u) \geqslant$ $-\psi(t)$ for all $(t, y, u) \in S$.

( $\beta)$ There are $\psi(t) \geqslant 0, t \in G, \psi \in L_{1}\left(G, E^{1}\right)$ and a constant $b \geqslant 0$ such that $f_{0}(t, y, u) \geqslant-\psi(t)-b\|f(t, y, u)\|_{B}$ for all $(t, y, u) \in S$.

Obviously, $(\psi)$ implies $(\alpha)$ (and hence also $(\beta)$ ), since from $(\psi)$ for $\epsilon=1$ we derive $f_{0} \geqslant-\psi_{1}(t)$. Condition $(\psi)$ is certainly satisfied under usual growth conditions, as for instance:

(g) Given $\epsilon>0$, there are $N \geqslant 0, P \geqslant 0$ such that, for all $(t, y, u) \in M$, we have

$$
\begin{gathered}
\|f(t, y, u)\|_{B} \leqslant P \quad \text { if } \quad\|u\|_{U} \leqslant N, \\
\|f(t, y, u)\|_{B} \leqslant \epsilon f_{0}(t, y, u) \quad \text { if } \quad\|u\|_{U}>N .
\end{gathered}
$$

Conditions (P12) shall now be replaced by the following hypotheses:

( $\left.\tilde{\mathbf{P}}_{1}\right)$ There is a bounded $\mu$-measurable function $\tilde{p}(t)=$ $\left(p^{0}(t), p(t)\right), t \in G, \tilde{p}: G \rightarrow E^{1} \times B$, say $\left|p^{0}(t)\right|,\|p(t)\|_{B} \leqslant \sigma$, such that for every $t \in G-T_{0}$ we have $\tilde{p}(t) \in \widetilde{Q}(t, y(t))$ for all $y \in A(t)$.

$(\tilde{\mathrm{P}} 2) \quad$ There are constants $c \geqslant 0, d \geqslant 0$ such that $z^{0} \geqslant-c$ for all $\left(z^{0}, z\right) \in \check{Q}(t, y)$ with $\|z\|_{B} \leqslant d$.

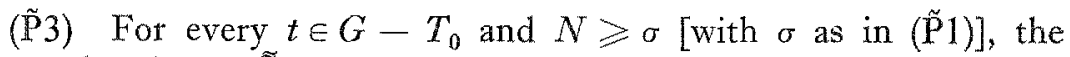
convex closed sets $\widetilde{Q}(t, y) \cap\left[E^{1} \times V(0, N)\right]$ satisfy property (Q) with respect to $y$ in $A(t)$.

Property ( $\mathrm{P} 1)$ is certainly satisfied if, for instance, the sets $U(t, y)$ contain the origin of $U$ and $f_{0}(t, y, 0)=0, f(t, y, 0)=0$. Then, property $(\tilde{P} 2)$ is also satisfied if $f_{0}$ is bounded below in $S$. Concerning ( $(\tilde{\mathrm{P}} 3)$, we only note that, for $B=E^{r}, Y=E^{s}, U=E^{m}$, the sets

$$
\widetilde{Q}(t, y) \cap\left[E^{1} \times V(0, N)\right], \quad y \in A(t),
$$


are convex, closed, and contained in the fixed cylinder $\left[\left(z^{0}, z\right)\left|z^{0} \leq E^{1},\right|\right.$ $|z| \leqslant N]$, and then the property $(\mathrm{Q})$ is certainly satisfied provided $|f(t, y, u)| \rightarrow+\infty$ as $|u| \rightarrow \infty$ uniformly on any bounded subsets of $A(t)$ [as a consequence of the mere continuity of $f_{0}, f$ on $S(t)$ ].

3.2. Existence Statement for Lagrange Problems, Let $A, S, f, f_{0}$ satisfy conditions (C) and $(\alpha)$. Let us assume that $\Omega$ is not empty and closed, and that $\{x\}_{\Omega}$ is sequentially relatively compact in $(X, \tau)$. Let us assume that both operators $L$ and $M$ have the closure property in $X_{0}$, and that at least one of them has the closed graph property in $X_{0}$. Then, the function $I[x, u]$ has an absolute minimum in $\Omega$ provided any of the following alternate assumptions holds.

Whenever it is known that $I[x, u]$ is bounded below in $\Omega$, or that $f$ has the weak convergence property, then condition $(\beta)$ can be required instead of $(\alpha)$.

\section{Alternate Hypotheses}

(AQ̃) The same hypotheses on $L$ and $M$ as in (AQ) of Section 2.4 and, for all $t \in G-T_{0}$, the sets $\widetilde{Q}(t, y)$ have property (Q) with respect to $y$ in $A(t)$ (in particular, these sets are convex and closed).

Alternately, we may assume that $f$ has weak convergence property in $L_{1}(G, B)$, and then (ii) can be deleted. The condition of $f$ is certainly satisfied if $f_{0}, f$ satisfy growth condition $(\psi)$ and property (R3) holds for $G, B$.

(A $\tilde{K})$ The same assumptions on $L, M$ as in (AK) of Section 2.4. For every $t \in G-T_{0}$, the sets $\widetilde{Q}(t, y)$ have property $(\mathrm{K})$ with $y$ in $A(t)$. The same alternate condition holds as in (AK).

$\left(\mathrm{AQ}{ }^{*}\right)$ The same assumptions on $L=L_{1} \times L_{2}, M, B=B_{1} \times B_{2}$, $f$ as in (AQ*) of Section 2.4. For every $t \in G \ldots T_{0}$ the sets $\mathscr{Q}(t, y)$ are now supposed to have property $\left(\mathrm{Q}^{*}\right)$ with respect to $y$ in $A(t)$ and $\left(E^{1} \times B_{1}, B_{2}\right)$ as in $\left(\mathrm{AQ}^{*}\right)$ of Section 2.3 . The same alternate condition holds as in $\left(\mathrm{AQ}^{*}\right)$.

(A $\left.\tilde{Q}^{* *}\right)$ The same as $\left(\mathrm{AQ}^{*}\right)$ with $L, M, B, f$ as in $\left(\mathrm{AQ}^{* *}\right)$ of Section 2.4 , and the sets $\widetilde{Q}(t, y)$ satisfying property $\left(\mathrm{Q}^{*}\right)$ with respect to $y$ in $A(t)$ and $\left(E^{1} \times B_{1} \times B_{2}, B_{3} \times B_{4}\right)$. The condition on $f_{1}$ is certainly verified if we assume that $f_{0}, f_{1}$ satisfy growth condition $(\psi)$ and $G, B_{1}$ satisfy (R3).

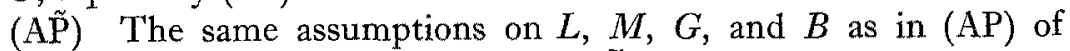
Section 2.4. We assume now that the sets $\widetilde{Q}(t, y)$ are convex and closed, and have geometric properties $(\tilde{\mathrm{P}} 1),(\tilde{\mathrm{P}} 2),(\tilde{\mathrm{P}} 3)$. The same alternate condition holds as in (AQ̃). 
$\left(\mathrm{A} \tilde{\mathrm{P}}^{*}\right)$ The same as in $\left(\mathrm{AP}^{*}\right)$ of Section 2.4 . The sets $\tilde{Q}(t, y)$ are

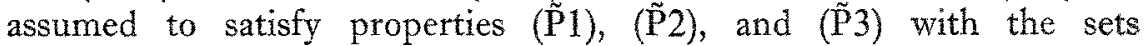
$\tilde{Q}(t, y) \cap\left[E^{1} \times V(0, N)\right]$ satisfying property $\left(\mathrm{Q}^{*}\right)$ with respect to $y$ in $A(t)$ relatively to $\left(E^{1} \times B_{1}, B_{2}\right)$. The same alternate condition holds as in $(\mathrm{A} \tilde{\mathrm{Q}})$.

$\left(\mathrm{A} \tilde{\mathrm{P}}^{* *}\right)$ The same as in $(\mathrm{AP} * *)$ of Section 2.4 . The sets $\tilde{Q}(t, y)$ are

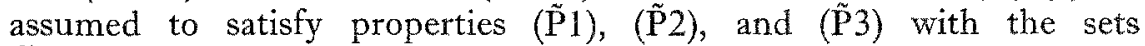
$\widetilde{Q}(t, y) \cap\left[E^{\mathbf{1}} \times V(0, N)\right]$ satisfying property $\left(\mathrm{Q}^{*}\right)$ with respect to $y$ in $A(t)$ relatively to $\left(E^{\mathbf{l}} \times B_{1} \times B_{2}, B_{3} \times B_{4}\right)$.

Conditions $\left(\tilde{\mathrm{F}}_{p}\right),\left(\tilde{\mathrm{G}}_{p q}\right),\left(\tilde{\mathrm{H}}_{q}\right)$. The same as $\left(\mathrm{F}_{p}\right),\left(\mathrm{G}_{p q}\right),\left(\mathrm{H}_{q}\right)$ of Section 2.4 , where now the sets $U(t)$ depend only on $t$, the sets $\tilde{Q}(t, y)$ are closed and convex, and both $f_{0}, f$ satisfy the analytic conditions stated in $\left(\mathrm{F}_{p}\right),\left(\mathrm{G}_{p q}\right),\left(\mathrm{H}_{q}\right)$, respectively.

3.3. Case of $f$ Linear and $f_{0}$ Convex in $u_{*}$ We assume here that $U(t)$ depends on $t$ only, that $B, Y, U$ are Banach spaces, that $f_{0}(t, y, u)$ is convex in $u$ for every $(t, y) \in A$, and that $f(t, y, u)=C(t, y) u+D(t, y)$ is linear in $u$, with $C, D$ as in Section 2.5 .

Statement 3.2 still holds in the present situation, with $f$ satisfying $\left(\mathrm{C}^{\prime}\right)$ and $f_{0}$ satisfying $(\mathrm{C})$, and the correspondent alternate assumptions $\left[f_{0}\right.$ satisfying $(\Gamma)$ and $f$ satisfying $\left(\Gamma^{\prime}\right)$ whenever needed, and both $f_{0}$ and $f$ satisfying $\left(\mathrm{G}_{p q}^{\prime}\right)$ whenever needed].

In the present situation, more alternate assumptions can be form mulated, as follows:

$\left(\tilde{\mathrm{G}}_{p q}^{\prime \prime}\right) 1<p, q \leqslant \infty$. The same as $\left(\mathrm{G}_{p q}^{\prime \prime}\right)$ of Section 2.5 , where $f_{0}$ is convex in $u, f$ is linear in $u ; f_{0}$ satisfies $(\mathrm{C}),(T)$, and the analytic conditions in $\left(G_{p q}\right) ; f$ satisfies $\left(\mathrm{C}^{\prime}\right),\left(\Gamma^{\prime}\right)$, and the analytic conditions.

Note that here the conditions imply that $f$ has the weak convergence property in $L_{1}(G, B)$. Thus, we did not need condition $(\psi)$ to guarantee this property.

3.4. Proof of Statements 3.2 and 3.3. The proof is the same as for Sections 2.4 and 2.5, where used is made of the lower closure theorems proved in the same papers (Refs. 8, 9, 11) mentioned in Sections 2.6.

\section{References}

1. Cesari, L., Existence Theorems for Problems of Optimization with Distributed and Boundary Controls, Proceedings of the International Congress of Mathematicians, Nice, France, Vol.3, pp. 157-161, 1970. 
2. Cesari, L., Closure Theorems for Orientor Fields and Weak Convergence, Archive for Rational Mechanics and Analysis, Vol 55, 1974.

3. CESARI, L., Existence Theorems for Weak and Usual Solutions in Lagrange Problems with Unilateral Constraints, $I, I I$, Transactions of the American Mathematical Society, Vol. 124, pp. 369-412 and 413-429, 1966.

4. Cesari, L., Closure Theorems for Orientor Fields, Bulletin of the American Mathematical Society, Vol. 79, pp. 684-689, 1973.

5. Cesari, L., and Cowles, D. E., Existence Theorems for Optimization Problems with Distributed and Boundary Controls, Archive for Rational Mechanics and Analysis, Vol. 46, pp. 321-355, 1972.

6. Cessari, L., and KaIser, P. J., Closed Operators and Existence Theorems in Multidimensional Problems of Calculus of Variations, Bulletin of the American Mathematical Society, Vol. 80, pp. 473-478, 1974.

7. Kaiser, P. J., Seminormality Properties of Convex Sets, Circolo Matematico di Palermo (to appear).

8. Kaiser, P. J., and Suryanarayana, M. B., Orientor Field Equations in Banach Spaces, Journal of Optimization Theory and Applications (to appear).

9. CesarI, L., Lower Semicontinuity and Lower Closure Theorems without Seminormality Conditions. Annali di Matematica Pura e Applicata, Vol. 98, pp. 381-397, 1974.

10. Cesari, L., A Necessary and Sufficient Condition for Lower Semicontinuity, Bulletin of the American Mathematical Society, Vol. 80, pp. 467-472, 1974.

11. Cesari, L, and Suryanarayana, M. B., Closure Theorems Without Seminormality Conditions, Journal of Optimization Theory and Applications (to appear).

12. Cesari, L., and Suryanarayana, M. B., Convexity and Property $(Q)$ in Optimal Control Theory, SIAM Journal on Control, Vol. 12, No. 4, 1974.

13. Berkovitz, L. D., Existence and Lower Closure Theorems for Abstract Control Problems, SIAM Journal on Control, Vol. 12, pp. 27-42, 1974.

14. Cesari, L., An Existence Theorem Without Convexity Conditions, SIAM Journal on Control, Vol. 12, No. 3, 1974.

15. Suryanarayana, M. B., Linear Control Problems with Total Differential Equations, Transactions of the American Mathernatical Society (to appear). 